\title{
DEL INFRARREALISMO AL REAL VISCERALISMO: BOLAÑO Y LA AUTOCRÍTICA DE UN MARGINAL ${ }^{1}$ \\ From Infrarrealismo to Real Visceralismo: Bolaño and the Self-Criticism of an Outcast
}

Ainhoa Vásquez Mejías*

Resumen

El artículo indaga acerca de la poética del movimiento mexicano "Infrarrealista", así como sus principales influencias estéticas y literarias, tanto nacionales como internacionales. Para ello se analiza la novela de Roberto Bolaño: Los detectives salvajes, como una reescritura autocrítica que realiza el autor a su propio proyecto poético varios años después de que este fracasara en su intento por aliar arte y vida.

Palabras clave: Infrarrealismo, Estética, Reescritura, Autocrítica, Fracaso.

Abstract

This article investigates the poetry of the Mexican movement "Infrarealism", as well as its main aesthetics and literary influences, both national and international. In order to do so, Roberto Bolaño's novel, The savage detectives, is analyzed as an selfcritical rewriting realized by the author to his own poetic project, several years after its failure in his attempt to fuse art and life.

Key words: Infrarealism, Aesthetics, Re-write, Self-criticism, Failure.

\section{INTRODUCCIÓN}

Roberto Bolaño llegó a México cuando apenas contaba con quince años de edad. Al poco tiempo decidió abandonar la escuela y dedicarse a leer y escribir, las dos cosas que más lo apasionaban; de esta forma, creció alejado de los circuitos oficiales de cultura y educación, convirtiéndose en un autodidacta. Durante muchos años no hizo más que vagar por un Distrito Federal del que no quiso ser parte a nivel académico, pero que le permitió realizar sus actividades favoritas: siempre había una librería en la que el vendedor distraído le permitiría robarse un buen libro, siempre había un parque donde sentarse a leerlo o donde mirar pasar a la gente. Así, Bolaño aprovechó el tiempo libre que tenía estudiando, pero también ideando su proyecto literario: qué tipo de poesía y de literatura era la que él quería hacer, en un mundo que ya no percibía o sentía como el suyo.

\footnotetext{
${ }^{1}$ Este artículo forma parte de la tesis con que obtuve el grado de Magíster en Literatura Latinoamericana por la Universidad Nacional Autónoma de México, con el apoyo tutorial del profesor Manuel Garrido.
} 
Años después encontró en su camino a otros escritores con ideales poéticos similares. Estos, sin embargo, diferían de él, porque pertenecían a la vida estudiantil. Casi todos eran alumnos de alguna carrera en la Universidad Nacional Autónoma de México y varios, además, eran miembros del taller de poesía a cargo de Juan Bañuelos o Julio César Álamo, como consta en Los detectives salvajes. Esa amistad que generó con ellos, lo llevaría más tarde a fundar un movimiento poético: los Infrarrealistas (en los años setenta) o real visceralistas (desde su relectura crítica en los años noventa). Será la novela Los detectives salvajes la encargada de plasmar, revisitar y autocriticar a esta corriente literaria generada a sexo, sudor, lágrimas y poesía. Intentaremos en el presente artículo vislumbrar los puntos centrales del infrarrealismo de los setenta y descubrir las estrategias desde las que se reviste para terminar transformado en "real visceralismo" la novela de fines de los noventa.

En cuanto a los infrarrealistas y sus inicios, sabemos que la mayoría de los futuros miembros del movimiento provienen del taller de Bañuelos, quienes de un día para otro deciden abandonarlo para fundar su propia perspectiva literaria. Patricia Espinosa señala al respecto: "El movimiento infrarrealista surge entre fines de 1975 y comienzos de 1976, en México DF, y lo conforman Mario Santiago, Ramón Méndez y Héctor Apolinar, que venían del fracasado taller de poesía de Difusión Cultural de la UNAM, coordinado por el poeta y académico Juan Bañuelos" (Espinosa, 2005).

Esta experiencia ha quedado plasmada en la novela desde la visión de un joven estudiante del primer semestre de Derecho que pronto engrosará las listas del nuevo movimiento: Juan García Madero. En la primera parte de la novela, escrita como diario del poeta advenedizo, comenta que un día aparecieron por el taller dos real visceralistas, quienes "pusieron en entredicho el sistema crítico que manejaba Álamo; quien, a su vez, trató a los real visceralistas de surrealistas de pacotilla y de falsos marxistas, siendo apoyado en el embate por cinco miembros del taller" (15). La batalla se pone ardua, todos se critican entre sí armando un gran escándalo. Sin embargo, todo culmina en el momento en que Arturo Belano (o Roberto Bolaño en su correlato infrarrealista) se levanta promulgando la búsqueda de poetas que quieran participar en la revista que pretenden editar los real visceralistas. García Madero acepta, comenzando así su trayectoria literaria y vivencial.

De esta forma comienza a constituirse el movimiento infrarrealista, cuyos principales exponentes fueron el chileno Roberto Bolaño y el mexicano Mario Santiago Papasquiaro (Ulises Lima en la novela), quienes en ese momento contaban con 23 años. Ellos se encontraron por primera vez en 1975 en el Café La Habana, lugar que posteriormente sería el espacio de reunión de los poetas infrarrealistas: "Allí, Mario Santiago le entrega a Bolaño un fajo de poemas que el chileno lee durante toda la noche" (Cobas, 12). Fue entonces cuando pocos meses después, una noche en casa del poeta Bruno Montané se gesta esta nueva corriente literaria, que 
termina por incluir tanto a escritores como músicos y pintores ${ }^{2}$. Los lugares de encuentro del real visceralismo, mencionados en la novela por García Madero son el café Quito, el Bucareli, Encrucijada Veracruzana, la casa de María Font en la colonia Condesa, o en la casa de la pintora Catalina O'Hara, en la colonia Coyoacán.

Los lineamientos generales del movimiento Infrarrealista fueron expuestos en un manifiesto redactado por Roberto Bolaño, titulado "Déjenlo todo, nuevamente"3. La incitación básica es que los poetas salgan a la calle a buscar una nueva sensibilidad, que buceen en la conciencia del hombre para conmover a partir de ahí su cotidianeidad. La realidad está debajo de lo que podemos aprehender a simple vista, como deja ver la metáfora del nombre "Infrarrealista": los poetas infra son como los infra del espacio, cuerpos sin luz que existen pero no se vislumbran "planetas oscuros calentados desde adentro y en cuyo interior generan su vida propia independientemente de un exterior que no puede verlos" (Cobas, 20).

La clave de la metáfora se encuentra en la marginalidad. Los poetas infrarrealistas debían permanecer alejados de los circuitos oficiales de cultura, y declararse abiertamente enemigos de la burocracia y los espacios de poder. No estaban dispuestos a venderse a las grandes editoriales y creían fielmente que un poeta no solo debía escribir sino vivir como tal: por ello, en las reuniones nunca faltó el alcohol; "pasión descarnada e irrefrenable energía etílica" (Ruiz, 2005) debían ir de la mano. El movimiento no era solo poesía sino una actitud ante la vida: "Nuestra ética es la Revolución, nuestra estética ${ }^{4}$ la Vida: una-sola-cosa" (Bolaño, Déjenlo todo). El compromiso del escritor frente a su mundo debe reflejarse en la acción, no en las palabras.

El compromiso del escritor con la vida misma implica un proceso continuo de transformación personal, de autocreación, de vulnerar los límites establecidos por una sociedad represiva, es por ello que la constante embriaguez, en la que varios críticos coinciden, así como el hecho de ver la poesía en su inmediatez y

\footnotetext{
${ }^{2}$ La lista propuesta por Patricia Espinosa es la siguiente: Juan Esteban Harrington (¿García Madero?), Piel Divina, Cuauhtémoc Méndez, Oscar Altamirano, José Peguero, Pedro Damián, Elmer Santana, Ramón Méndez, Guadalupe Ochoa, Edgar Altamirano, Mará Larrosa, Vera Larrosa (¿las hermanas Font?), Kyra Calvan, Víctor Monjarás, Carlos David Marfarón, Geles Lebrija, Rubén Medina, José Rosas Ribeyro, Estela Ramírez, Lorena de la Rocha y Javier Suárez Mejía.

${ }^{3}$ La lectura del Primer Manifiesto Infrarrealista se llevó a cabo en una Librería Gandhi del D. F. en 1976. El título presenta reminiscencias claras al manifiesto Lanchez tout publicado por André Breton: "Dejad todo. Abandonad Dadá. Abandonad a vuestra mujer, a vuestra amante. Abandonad vuestras esperanzas y vuestros temores. Abandonad lo conocido por lo desconocido. Partid por los caminos" (De Torre, 17)

${ }^{4}$ Resulta interesante remitirnos a las definiciones propuestas por Kierkegaard sobre lo estético. Para el teórico Theodor W. Adorno, la estética en la obra del filósofo se relaciona directamente con lo fugaz, con la inmediatez, con la vida tal como se nos presenta: "Lo estético en el hombre es aquello por lo cual él es inmediatamente lo que él es: lo ético en él es aquello por lo cual se convierte en lo que se convierte. El que vive en y de lo estético, por y para lo estético en él, vive estéticamente" (Adorno, 23). De esta forma, podríamos relacionar la idea de estética de Roberto Bolaño con la del filósofo Kierkegaard, en cuanto a la existencia estética como pura inmediatez, la poesía como la vida misma.
} 
como un sinónimo de vida, puede deberse a la búsqueda de nuevos tipos de realidad. En este sentido, los infrarrealistas se valdrían de ciertos atributos relacionados a la poética del romanticismo y el surrealismo, aunque ambos movimientos de vanguardia se centran en la imaginación, en el sueño como mecanismo para llegar a las profundidades humanas, en el intento de bucear en la inconsciencia y lograr ver más allá de lo evidente, tal como señala Albert Beguin (1954) en su libro El alma romántica y el sueño, sobre la figura de Rimbaud: "el poeta es un vidente, un visionario; llega a lo desconocido, encuentra lo nuevo"(483). De la misma manera, el escritor surrealista "se abandona por entero a las fuerzas oscuras de lo inconsciente, hace por provocarlas; escarba en su interior con el propósito de aflorar el oro y la escoria" (De Torre, 28). No conformarse con lo aparente, dejarse caer en el abismo, aliar vida y $\operatorname{arte}^{5}$, atributos entre románticos y vanguardistas que Bolaño retomará en su propio proyecto.

Ese espíritu es el que se plasma en Los detectives salvajes: los real visceralistas son marginales por opción propia, alejados de los circuitos de consagración, deambulan por el Distrito Federal sin dinero para tomar el camión, al margen de las instituciones: apenas García Madero se une al movimiento, deja de ir a la universidad: "Hoy no fui a la universidad. He pasado todo el día encerrado en mi habitación escribiendo poemas" (17); la revista Lee Harvey Oswald publicada y financiada por Ulises Lima deja de funcionar cuando otros la reconocen: "Lee Harvey Oswald debió continuar, la cortaron justo en el mejor momento, cuando la gente empezaba a conocernos" (31), dice Pancho Rodríguez.

La gente que los rodea también remarca la característica de marginalidad de los poetas; mientras Alfonso Pérez Camarga, asegura que Belano y Lima no eran revolucionarios ni escritores ni poetas, sino simples vendedores de droga, Carlos Monsiváis (personaje en la ficción), señala que tan marginales son que no tuvieron dinero ni siquiera para pagar un café: "En algún momento de debilidad (mental, supongo), me recordaron a José Agustín, a Gustavo Sainz, pero sin el talento de nuestros dos excepcionales novelistas, en realidad sin nada de nada, ni dinero para pagar los cafés que nos tomamos (los tuve que pagar yo), ni argumentos de peso, ni originalidad en sus planteamientos. Dos perdidos, dos extraviados" (160).

De esta forma, los real visceralistas viven tal como se lo han propuesto en el manifiesto Infrarrealista: como poetas ligados al acontecer histórico, a la vida cotidiana. Tal es su estética. Sus preocupaciones éticas, en cambio, van mucho más allá de tener dinero para comprar un café o tener que levantarse día a día para ir a una universidad que nada aportará en un futuro, al menos no en el futuro que ellos buscan.

\footnotetext{
${ }^{5}$ Octavio Paz (1998) concluye que la semejanza central entre el romanticismo y el surrealismo (nosotros agregamos el infrarrealismo): "es la pretensión de unir vida y arte. Como el romanticismo, la vanguardia no fue únicamente una estética y un lenguaje; fue una erótica, una política, una visión del mundo, una acción: un estilo de vida" (148).
} 
Por esta misma razón, no se identifican con ninguno de los grupos poéticos ni políticos que pululan por Latinoamérica en esos momentos:

No estaban en ninguno de los dos bandos (el de Octavio Paz y el de los poetas campesinos), ni con los neopriístas ni con la otredad, ni con los neoestalinistas ni con los exquisitos, ni con los que vivían del erario público ni con los que vivían de la Universidad, ni con los que se vendían ni con los que compraban, ni con los que estaban en la tradición ni con los que convertían la ignorancia en arrogancia (352).

La poesía y la vida de los infrarrealistas, como lo expresa lúcidamente el más loco de la novela, Joaquín Font, era para los desesperados, por ello la insistencia, tanto en "Déjenlo todo, nuevamente", como en la misma novela Los detectives salvajes, en la idea de romper con la cultura dominante, vendida a las industrias, alejada de la realidad, y recuperar la función primigenia del poeta: observar y cambiar esa visión desde la estética, la ética de la revolución. Dentro de este pensamiento, queda clara la oposición tajante al grupo de Octavio Paz y "los poetas campesinos".

El mismo Manifiesto Infrarrealista se planteó desde sus orígenes como una reacción en contra de la cultura establecida y en decadencia, que se reflejaba tanto en los medios de prensa y en las fundaciones como en los circuitos literarios. Es por ello que una de las propuestas principales fue "volarle la tapa de los sesos a la cultura oficial" (Bolaño, Déjenlo todo); una cultura que veía en la imagen de Octavio Paz a su mayor exponente, y quien contaba con una tropa de seguidores apodados "poetas estatales", porque se creía que el Partido Revolucionario Institucional les pagaba por su concordancia política. Así, "los infrarrealistas se volvieron contra el fundador de la revista Plural porque representaba todo aquello que odiaban, una intelectualidad a la que le daba lo mismo servir o no de conciencia a la clase dominante" (Sánchez, en línea).

En la novela también existe una crítica severa al poeta Octavio Paz, muchas veces se llega a decir que es "nuestro enemigo". Es así como no podemos dejar de notar la oposición constante que se realiza en el libro entre aquellos poetas marginales, como son los real visceralistas y los poetas estatales, que escriben para el gobierno o para el mercado editorial. Entran en franco conflicto las dos posturas: los jóvenes poetas, rebeldes y desesperados que no tienen exactamente un grupo literario sino una "pandilla", que no saben de retórica o figuras poéticas, que no están dispuestos a ingresar al mercado editorial y que hartos ya del imperio de Neruda y $\mathrm{Paz}^{6}$, pretenden revolucionar la poesía latinoamericana; en contraposición a los poetas campesinos, que publican constantemente, se hacen llamar parte de un movimiento

6 “Coincidimos plenamente en que hay que cambiar la poesía mexicana. Nuestra situación (según me pareció entender) es insostenible, entre el imperio de Octavio Paz y el imperio de Pablo Neruda. Es decir: entre la espada y la pared" (Bolaño, Los detectives, 30). 
poético, son cultos en retórica y palabrería inútil $\mathrm{y}$, como si ello fuera poco, no presentan intenciones de cambiar nada.

$\mathrm{Y}$ es que lo que proponía Bolaño en su Manifiesto es que el poeta debe comprometerse con la vida y con el momento histórico que le corresponde; basta de intelectuales en sus burbujas de poder; el verdadero poeta es quien es capaz de dejarlo todo y "lanzarse a los caminos". Es por esta razón que junto al tópico recurrente de la marginalidad se sumará para complementarlo el de la errancia, el vagabundeo, que ante todo llama a no quedarse en un mismo sitio a ver pasar los acontecimientos, sino unirse a ellos ${ }^{7}$. Será por este motivo que los detectives salvajes irán tras la pista de Cesárea Tinajero (madre del real visceralismo) al desierto de Sonora; viaje iniciático que pronto los llevará aún más lejos, en busca de su propio destino.

Cesárea Tinajero proviene de un movimiento literario inspirador del real visceralismo: el estridentismo, cuyos principales representantes fueron Manuel Maples Arce (ideólogo), Germán List Arzubide y Salvador Gallardo, entre otros. Dicho grupo surge influenciado por dos acontecimientos mundiales: la Revolución mexicana y la Revolución rusa; por ello, no es de extrañar que se autopromulgaran: "el primer movimiento revolucionario-literario-social" (Schwartz, 2002), en su intento por aliar estética con revolución. En Los detectives salvajes Amadeo Salvatierra (correlato de Maples Arce) rememora el pasado estridentista en el que pretendían "hacer de la literatura una práctica capaz de dar cuenta de un presente poblado de máquinas, carteles publicitarios y arcos voltaicos" (Cobas y Garibotto, 164), apoyada dicha idea en las estructuras de poder revolucionario, que en los años veinte se encontraba en proceso de institucionalización.

Vemos así que al igual que la corriente Infrarrealista y su correlato novelado, este grupo presenta la intención de unir revolución y vida, una revolución que solo será posible mediante la modernización y las máquinas. Este punto es de especial relevancia, por cuanto si bien la temática de la novela de Bolaño es inminentemente urbana, la ciudad ya no está idealizada, ellos han llegado a la pérdida de esa ilusión de la máquina como fuente de revolución ${ }^{8}$. Los infrarrealistas se han dado cuenta de que el progreso es una fantasía, la ciudad se presenta como el fracaso de los grandes ideales modernos, el desencanto de lo que no fue. Así, reconocen que la revolución no debe hacerse mediante la tecnología, sino con poesía, en la alianza entre arte y vida. El cosmopolitismo que promulgaban los estridentistas da paso al desplazamiento de

\footnotetext{
${ }^{7} \mathrm{Si}$ bien el movimiento infrarrealista y su correlato real visceralista presentan ciertas semejanzas con el movimiento beatnik, en cuanto a la pasión etílica, el deambular constante y la exaltación de los sentidos, se diferencia de manera sustancial con el grupo de Roberto Bolaño, ya que la generación beat se define desde la rebeldía sin causa y sin consecuencias y no presentan un proyecto estético determinado (De Torre, 1971).

${ }^{8}$ Como señala Octavio Paz, los jóvenes de los setenta ya no ven a las grandes ciudades desde las utopías de progreso y civilización, sino: "Los hombres empiezan a ver con terror el porvenir y lo que apenas ayer parecían las maravillas del progreso hoy son sus desastres. El futuro ya no es el depositario de la perfección, sino del horror" (213).
} 
los infrarrealistas: la ciudad no acoge, no cobija, no presenta una esperanza de cambio y bienestar.

Si bien la poesía no es ya plenamente estridentista, porque ha inventado su propio movimiento del que los real visceralistas solo son los herederos, el proyecto siempre es el mismo: "Todos los mexicanos somos más real visceralistas que estridentistas, pero qué importa, el estridentismo y el realismo visceral son solo dos máscaras para llegar a donde de verdad queremos llegar. ¿Y adónde queremos llegar?, dijo ella. A la modernidad, Cesárea, le dije, a la pinche modernidad" (Bolaño, Los detectives, 460). Ante este fracaso de la modernidad promulgada tanto por los estridentistas como por Tinajero, la única alternativa es "lanzarse a los caminos", dejando la gran ciudad en busca de los restos del naufragio.

Es así como llegarán al desierto de Sonora, donde encontrarán a la madre del real visceralismo en condiciones completamente diferentes a las que ellos imaginaron. La derrota de los ideales se hace aún más patente en la figura desgastada de la poeta: una mujer gorda que ha dejado de escribir, ni rastros de la bella mujer que alguna vez fue. De esta forma, la figura de Tinajero "condensa el fracaso del proceso de modernización emprendido por la vanguardia en los 20 . Con su pasaje del sueño de la gran urbe moderna al desierto de Sonora, Cesárea rompe a un tiempo con la vanguardia y con la revolución. Este es el momento en el que, como recuerda Salvatierra, todo comienza a deslizarse irremediablemente por el precipicio" (Cobas y Garibotto, 167).

Cobas y Garibotto proseguirán señalando que el encuentro con Cesárea y su posterior deceso no hace más que constatar la derrota del primer real visceralismo comprobando, al mismo tiempo, la del segundo. Tinajero simbolizará, así, el fracaso del proyecto poético modernizador y el intento de aliar literatura, revolución y vida. Su muerte implicará la desintegración del segundo movimiento, la muerte de la poeta representará el punto cúlmine de una generación en decadencia: "Oí que Belano decía que la habíamos cagado, que habíamos encontrado a Cesárea solo para traerle la muerte" (Bolaño, Los detectives, 605). Luego de este hecho, los integrantes del segundo real visceralismo deben huir, Lima y Belano se separarán en Europa: cada uno intentará encontrar su propio destino, será así, el fin del proyecto moderno de colectividad.

De esta manera Bolaño y los infrarrealistas encarnan a un grupo de desencantados, quienes buscaban la concordancia entre el ser y el hacer del poeta, marginales de vocación, sin confianza en nadie, jóvenes que soñaban "con utopía y se despertaron gritando" (Bolaño, Déjenlo todo), tal como el escritor anuncia en el Manifiesto. Frente a un mundo adverso solo les quedaba la provocación y el insulto, "perros rabiosos, perros apaleados" (Herralde, 25) como señaló en alguna ocasión su amigo y editor Jorge Herralde. Desesperados, marginales, miembros activos del club de la desesperanza, boicoteadores de los actos oficiales, finalmente, "verdaderos poetas" como escribe Bolaño en su poema Como una vieja balada 
anarquista: "los verdaderos poetas tiernísimos/ metiéndose siempre en los cataclismos más atroces/ más maravillosos/ sin importarles/ quemar su inspiración/ sino donándola/ sino regalándola/ como quien tira piedras y flores" (Bolaño, Muchachos desnudos).

Juan Villoro confirma lo señalado y agrega: "Bolaño escribe sobre poetas que indagan el reverso de las cosas y transforman la experiencia en obra de arte [...] Los poetas de Bolaño viven la acción como una estética de vanguardia. Algunos de ellos escriben cosas que no leemos, otros buscan la gramática del desmadre, todos resisten" (Herralde, 16). Finalmente, lo que más les importaba a los infrarrealistas no era el hecho de escribir poesía, sino vivir como poeta, la vida cotidiana era el poema mismo: "Mi interés básico era ese, vivir como poeta. Para mí, ser poeta era, al mismo tiempo, ser revolucionario y estar totalmente abierto a cualquier manifestación cultural, a cualquier expresión sexual [...]. La tolerancia era... Más que tolerancia, palabra que no nos gustaba mucho, era hermandad universal" (Braithwaite, 38).

$\mathrm{Y}$ es ese proyecto el que muere junto a Cesárea Tinajero. La hermandad se diluye y los real visceralistas desaparecen: "Lupe me ha dicho que somos los últimos real visceralistas que quedan en México" (Bolaño, Los detectives, 606). O como expresa Rafael Barrios, una vez que Lima y Belano se van a Europa, los demás integrantes del movimiento comienzan a hacer performances, escritura masturbatoria, teatro del absurdo, haikús, epigramas: "Hicimos lo que pudimos... Pero nada salió bien" (214). Por otra parte, Xóchitl intenta encontrar trabajo, publicar sus poemas, sin embargo, por haber estado ligada a dicha poética, le responden negativamente. Finalmente, el estudioso de Pachuca nos ofrece algunos datos sobre el paradero de otros: San Epifanio murió, Rafael Barrios desapareció en EE.UU., Piel Divina murió, Pancho Rodríguez murió, Emma Méndez se suicidó, Juan García Madero se desvanece como si nunca hubiese existido.

A la luz de lo revisado hasta el momento, queda claro que Los detectives salvajes, mientras que por una parte nos otorga luces para desentrañar la figura de Roberto Bolaño dentro de la literatura mexicana del siglo XX; por otra nos remite a la marginalidad de escritores jóvenes, influenciados por los procesos políticos revolucionarios de los años setenta y que finalmente terminan en el desencanto, en la frustración, en la batalla perdida. Poetas contestatarios, fuera de todo orden y alejados de los circuitos de poder y cultura oficial. Es por ello que años después es el mismo Roberto Bolaño quien expresa en la novela varios puntos con los que ya no concuerda. La historia y los personajes que en ella se relatan están escritos con la mirada de la experiencia... la autocrítica es el proceso lógico de quien busca también reencontrarse con su pasado.

La primera señal de dicha autocrítica la encontramos en el traslado del nombre del movimiento: el infrarrealismo da paso al real visceralismo. El infrarrealismo, como veíamos anteriormente, refiere a cuerpos sin luz o planetas 
oscuros que no todos pueden observar. Implica el sentirse distinto así como el llamado a ver más allá. Esa intención no cambiará de manera radical en la novela, sin embargo, con su rebautizo se agregará un cambio fundamental: real visceralismo remitirá ahora a esa visceralidad con que estos jóvenes poetas actuaban, a la irracionalidad y el ímpetu con que atacaban a los poetas oficiales, porque ellos mismos, en su orfandad, no tenían nada que perder. Y el primer término "real" no hace más que remitirnos al intento de hacer una poesía cercana a la cotidianeidad, ligada a la realidad; la idea de vivir como poetas al límite, diciendo todo, expresando todo, sintiendo todo, bebiendo todo: viviendo.

La autocrítica, por supuesto, irá más allá. La visión de la poesía y la vida infrarrealista ya no será tan seria; en su traslado al real visceralismo se tornará juego, complicidad de amigos. El mismo nombre del movimiento no tiene mayores problemas al verse alterado, los mismos integrantes lo cambian constantemente: "Me inscribí en el taller de poesía de Julio César Álamo, en la Facultad de Filosofía y Letras, y de esta manera conocí a los real visceralistas o viscerrealistas en incluso vicerrealistas como a veces gustan llamarse" (Bolaño, Los detectives, 13). El nombre mismo es un juego, una broma, no es un movimiento literario en cuanto tal, más bien, es una "pandilla". García Madero se vuelve un integrante del grupo sin saber qué es el real visceralismo, la misma confesión que realizará, años después, Rafael Barrios: la duda, la ignorancia respecto del nombre del grupo, a los intereses, a la poesía que deben hacer.

Algunos lineamientos generales se mantienen desde el infrarrealismo, pues como señala García Madero, en un momento recordó que "una de las premisas para escribir poesía preconizadas por el realismo visceral, [...] era la desconexión transitoria con cierto tipo de realidad" (19-20). Ese tipo de realidad es justamente la idea de sentir las cosas tal como estas vienen y no transformarlas a nuestro antojo. Tomar y vivir la vida tal como esta se nos presenta, con sus incongruencias, extrañezas o complejidades: un verdadero poeta es el hombre capaz de recibir la realidad, la cotidianeidad y "vivirla". Es por esto también la referencia a los poetas que caminan hacia atrás, mirando un punto pero alejándose de él en línea recta: un real visceralista debe ser capaz de transitar lo desconocido, porque ahí también hay realidad, no conformarse con lo reconocible, con lo dado, ir siempre en busca de lo que otros no ven o no quieren descubrir.

A pesar de que estos lineamientos o tópicos del infrarrealismo se perpetúan en el real visceralismo, la autocrítica prosigue. Se señala que estos poetas, en su afán de vivir como tal, no son más que una estafa. Muchos traen consigo libros en francés, inglés o alemán, pero reconocen que no saben leer ninguno de esos idiomas; hablan de poesía e invitan a García Madero a pertenecer al movimiento sin siquiera haber leído un poema suyo. O tal como los describe María Font, ninguno realmente lee, solo se limitan a robar los libros: 
- No me hagas reír. Pero si en ese grupo solo leen Ulises y su amiguito chileno. Los demás son una pandilla de analfabetos funcionales. Me parece que lo único que hacen en las librerías es robar libros.

-Pero después los leerán, ¿no? —concluí un poco amoscado-.

-No, te equivocas, después se los regalan a Ulises y a Belano. Estos los leen, se los cuentan y ellos van por ahí presumiendo que han leído a Queneau, por ejemplo, cuando la verdad es que se han limitado a robar un libro de Queneau, no a leerlo (56).

Por otra parte, la ácida crítica que los infrarrealistas realizaron a Octavio Paz (interrumpiendo en sus actos públicos, planeando su secuestro, insultándolo abiertamente, etc.), en la novela se encuentra ya matizada y criticada por otros poetas. Luis Sebastián Rosado se declara en desacuerdo con los poetas, por cuanto él sí cree en lo que hace el poeta oficial. Y al decir esto se burla de los real visceralistas, argumentando que a ellos solo parecía gustarle lo que hacían ellos mismos. Posteriormente, Carlos Monsiváis (personaje de la novela) también los criticará por esta misma actitud que él califica de infantil: "obstinados en no reconocerle a Paz ningún mérito, con una terquedad infantil, no me gusta porque no me gusta, capaces de negar lo evidente" (160).

Y más importante aún que la burla que realizan algunos poetas por esa actitud de crítica infantil frente a Octavio Paz, resulta el episodio del encuentro entre el poeta oficial y Ulises Lima en el Parque Hundido. Será en ese momento cuando Roberto Bolaño, poeta Infrarrealista, haga las paces simbólicamente con el Premio Nobel. En un comienzo, Ulises lo observa cada día, hasta que la secretaria de Paz, Clara Cabeza, lo aborda para preguntarle quién es. Este responde sin problemas su nombre, agregando además que es el penúltimo poeta real visceralista que queda en México. Octavio Paz reconoce en el movimiento la participación de Cesárea Tinajero. Desde ya la sola mención de la poeta, el ambiente entre ellos se vuelve tranquilo, de reconciliación.

Ha llegado el momento en que ambos poetas dejen de lado sus diferencias. Ulises Lima ya no es el mismo niño, algo ha cambiado en esos años; Octavio Paz, por su parte, no guarda rencores en contra de él ni su movimiento. La conversación no dura mucho, sin embargo, Clara Cabeza señala que fue "distendida, serena, tolerante" (510). Poco después Lima se levanta y estrecha la mano de Paz. El encuentro es breve, pero deja clara la intención de reconciliar, al menos en la novela, a los jóvenes poetas marginales, con el consagrado escritor. Roberto Bolaño, 20 años después sabe que las viejas rencillas infantiles de nada servirán. La madurez del autor conlleva, en este caso, la autocrítica a su propia impulsiva juventud.

En una entrevista el mismo Bolaño se refiere a este episodio central: "En la novela cuando conversa (Octavio Paz) con Ulises Lima, hacen las paces. Se dicen todo lo que tienen que decirse, y eso, solo lo saben ellos. Así me imaginé a ese Paz viejo, ya con el Nobel, hablando el poeta joven. Paz todavía estaba vivo cuando escribí esto" (Posadas, 2008). Esa reconciliación será fundamental para entender lo 
que nos ha interesado rescatar a lo largo de este artículo: la autocrítica que se realiza años después al primer proyecto poético. Ese encuentro será en la novela el modo de asumir el fracaso de las utopías real visceralistas; sin embargo, en la obra del escritor será la forma de poner fin a la crítica y a la lucha que tantos años le realizó. Finalmente, todos los poetas, aunque den vueltas en direcciones opuestas (como Lima y Paz en el Parque Hundido), terminan encontrándose en el mismo círculo, parece haber demostrado el escritor en su paso por México y su inclusión en el movimiento infrarrealista.

\author{
Pontificia Universidad Católica de Chile* \\ Facultad de Letras \\ Seminario 61 depto. 1, Providencia (Chile) \\ ainhoavasquezm@gmail.com
}

\title{
OBRAS CITADAS
}

Adorno, Theodor. Kierkegaard: construcción de lo estético, Madrid: Akal, 2006.

Beguin, Albert. El alma romántica y el sueño: ensayo sobre el romanticismo alemán y la poesía francesa, México: Fondo de Cultura Económica, 1954.

Bolaño, Roberto. "Déjenlo todo, nuevamente. Primer Manifiesto Infrarrealista". Revista Viento en Vela, $\mathrm{N}^{0}$ 5, septiembre del 2006, México, D.F.

-----Los detectives salvajes, Barcelona: Anagrama, 1998.

------Muchachos desnudos bajo el arcoiris de fuego. México: Editorial Extemporáneos, 1979.

Braithwaite, Andrés (Selección y edición), Bolaño por sí mismo. Entrevistas escogidas, Santiago de Chile: Ediciones Universidad Diego Portales, 2006.

Cobas, Andrea. "La estupidez no es nuestro fuerte: Tres manifiestos del infrarrealismo mexicano". Osamayor. Graduate Student Review, Año XVII, $\mathrm{N}^{\circ}$ 17, University of Pittsburgh, 2006.

Cobas, Andrea y Garibotto, Verónica. "Un epitafio en el desierto: poesía y revolución en Los detectives salvajes", Bolaño salvaje, Edmundo Paz Soldán y Gustavo Faverón (eds.), Barcelona: Candaya, 2008.

De Torre, Guillermo. Historia de las literaturas de vanguardia, vol. III, Madrid: Guadarrama 1971.

Espinosa, Patricia. "Bolaño y el Manifiesto Infrarrealista". Rocinante, $\mathrm{N}^{\mathrm{o}}$ 84, octubre 2005.

Herralde, Jorge. Para Roberto Bolaño, México: Sexto Piso, 2005, p. 25.

Paz, Octavio. Los hijos del limo: del romanticismo a la vanguardia, Barcelona: Seix Barral, 1998. 
Posadas, Claudia. "La escritura salvaje de un nómada". <En línea $>$. Santiago, 2008. [Citado 11 de noviembre de 2009]. Disponible en: $\mathrm{http}: / /$ www.letras.s5.com/rb270208.html

Ruiz, Felipe. "Bolaño y el país de los soles negros". Artes y Letras de El Mercurio, domingo 6 de noviembre de 2005.

Sánchez, Matías. "El pasado Infrarrealista de Bolaño" <En línea $>$. Santiago, 27 de octubre del 2005. [Citado 11 de noviembre de 2009]. Disponible en: http://www.letras.s5.com/rb271005.htm

Schwartz, Jorge. Las vanguardias latinoamericanas: textos programáticos y críticos, México: Fondo de Cultura Económica, 2002. 